\title{
Central Projections of Identified Drosophila Sensory Neurons in Relation to their Time of Development
}

\author{
John Palka, Mark A. Malone, Richard L. Ellison, and Donald J. Wigston' \\ Department of Zoology, University of Washington, Seattle, Washington 98195
}

Eight sensory structures (campaniform sensilla), appcaring identical in the light and scanning electron microscopes, are found in specific locations on the wings of Drosophila. Their axons enter one of 2 central tracts, a medial one or a lateral one. The topographic arrangement of the sensilla on the wing is not reflected in this central projection pattern. There is, however, a strict correlation between the time when a sensillum develops and the path its axon follows: The 4 sensilla whose axons form the medial projection are born and differentiate early during the development of the wing, while the other 4 sensilla, all of which project laterally, arise during a second wave of differentiation. This time-related projection pattern remains stable in the face of a variety of genetically induced alterations in the precise number and location of sensilla.

The sensory systems of insects, like those of vertebrates, are frequently organized in topographic fashion (e.g., Johnson and Murphey, 1985; Murphey, 1981; Strausfeld, 1976). Because differentiation in a sensory field often proceeds in a topographically ordered way, there can be a simultaneous correlation between the position of a sensory element in the periphery, its age, and its central projection (Murphey et al., 1980; Walsh and Guillery, 1984).

We report here an analysis of the central projections of a set of 8 identified sensory elements in the wing of the fruitfly Drosophila melanogaster, whose cuticular components appear identical by light and scanning electron microscopy, and whose cell bodies normally differentiate in a sequence that does not correspond with their position. We find that in this system the region of the CNS to which a neuron projects is related to the time of its birth and differentiation, rather than to its peripheral position. This time-related axonal distribution pattern remains stable even when the numbers or locations of the neurons contributing to it are altered by genetic manipulation. A preliminary account of these results has been published (Wigston et al., 1984).

Ongoing studies indicate that the physiological properties of these receptors are not identical, in spite of the structural similarity of the cuticular elements, so that a three-way correlation between time of differentiation, central projection, and physiological function is established (Dickinson and Palka, 1985, and unpublished observations).

\footnotetext{
Received Oct. 2, 1985; revised Dec. 9, 1985; accepted Dec. 18, 1985.

We thank W. A. Harris, C. E. Holt, E. R. Macagno, R. K. Murphey, and members of our laboratory for commenting on the manuscript; Eric Cole for painstaking selection of useful genetic variants; Usha Rani for patient technical help; and especially Peter Kareiva for carrying out the statistical analysis. Supported by NIH Grant NS-07778 and NSF Grant 83-19890 to J.P. and an NIII Postdoctoral Fellowship to D.J.W.

Correspondence should be addressed to John Palka at Department of Zoology, NJ-15, University of Washington, Seattle, WA 98195.

I Present address: Department of Physiology, Emory University School of Medicine, Atlanta, GA 30322.
}

Copyright $\odot 1986$ Society for Neuroscience $0270-6474 / 86 / 061822-09 \$ 02.00 / 0$

\section{Materials and Methods}

Stocks

The wild-type strains Canton S, Oregon-R, and Sevelen were used; there were no obvious differences among them. Inbred lines with a single twin sensillum of the margin (TSM sensillum) were obtained by selection from the Sevelen stock; those with a distally shifted distal- (d-) TSM sensillum and a fourth third longitudinal vein (L3) sensillum were selected from an Oregon-R stock; and individuals with a proximally shifted anterior cross vein (ACV) sensillum were found in Oregon-R stocks (for a map of sensillar locations, see Fig. 1). The mutants vestigial and Hairy wing are described in Lindsley and Grell (1968).

\section{Staging}

As is conventional in studies of Drosophila development, developmental time was measurcd from the white prepupa stage, a 20 min period during which the third instar larva shortens and everts its spiracles, but before the larval cuticle starts to tan and form the puparium. Times are given as hours after pupariation (hr AP).

\section{Filling procedure}

In order to fill single axons with dye, individual sensilla were penetrated through their domes with a micropipette at $200-400 \times$ magnification under a fixed-stage compound microscope. Hexamine cobaltous chloride was then injected into the receptor lymph space (Thurm and Kuppers, 1980) with positive current pulses (10-20 nA on a $90 \%$ duty cycle for 4-10 min).

Even following silver intensification of the cobalt sulfide precipitate, the neuropil in these preparations appeared rather featureless, and it proved difficult to assign single filled axons to specific tracts. Therefore, we established a reference within the ganglion by also filling all the axons coming from the contralateral wing; the extreme symmetry of the projections from the 2 wings then permitted a reliable characterization of the path of the single axon. Whole-wing fills were made by puncturing vein L3 and applying a sliver of agar made up with $250 \mathrm{~mm}$ cobaltous chloride to this small wound $3 \mathrm{hr}$ after injection of individual sensilla. Ganglia were exposed, reacted with ammonium sulfide, and fixed in Carnoy's solution about $1 \mathrm{hr}$ later. Intensification was generally carried out the following day.

\section{Intensification}

We found the following procedure, a modification of Timm's sulfidesilver method (Bacon and Altman, 1977; R. Fricke, personal communication), to work well: Exposed ganglia were incubated in a dark oven at $50^{\circ} \mathrm{C}$ for $30 \mathrm{~min}$ in $50 \%$ solution $\mathrm{A}$ ( $3 \mathrm{gm}$ gum acacia, $10 \mathrm{gm}$ sucrose, and $0.43 \mathrm{gm}$ citric acid in $100 \mathrm{ml}$ distilled water); $30 \mathrm{~min}$ in $100 \%$ solution A; $90 \mathrm{~min}$ in solution B (solution A plus $0.17 \%$ hydroquinone) and 5-40 min in solution $C(2$ volumes of $1 \%$ silver nitrate plus 15 volumes of solution $B$ ). While in solution $C$, the specimens were removed briefly from the oven every few minutes and observed under a dissecting microscope, as the optimal intensification time was variable. Following intensification, the tissue was dehydrated in an alcohol series, rinsed in methyl salicylate, and mounted in neutralized Canada Balsam.

\section{Data analysis}

Fills of specific sensilla were accepted for analysis only if a single filled axon could be seen to enter into and branch within the CNS. We some- 


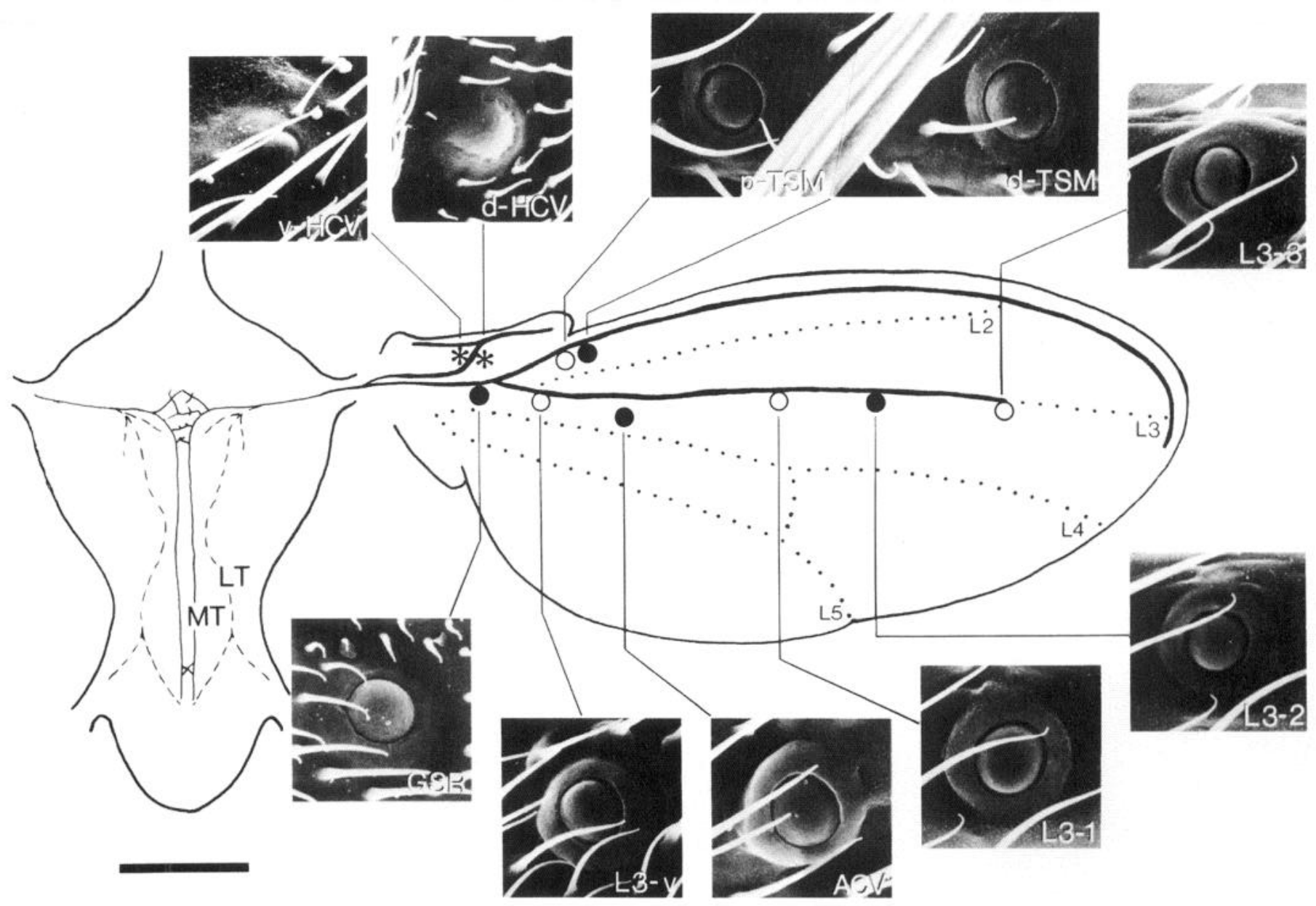

Figure 1. Campaniform sensilla on the wing of Drosophila melanogaster and the projections of their axons in the CNS. The scanning electron micrographs show the 8 virtually identical sensilla of the wingblade: the proximal and distal twin sensilla of the margin ( $p$ - and $d$-TSM), the 3 dorsal sensilla of the third longitudinal vein $(L 3-3, L 3-2$, and $L 3-1)$, the anterior cross vein sensillum $(A C V)$, the ventral sensillum of the third vein $(L 3-v)$, and the giant sensillum of the radius $(G S R)$. The d-TSM, L3-2, ACV, and GSR (closed circles) develop early and project into the medial tract (MT, solid line); the p-TSM, L3-3, L3-1, and L3-v develop later and project into the lateral tract (LT, dashed line). All these sensilla are in the anterior compartment (Garcia-Bellido et al., 1973), and all except the L3-v are dorsal. Proximally on the wing are found 2 additional early-developing sensilla of different morphology, the ventral and dorsal sensilla of the humeral cross vein ( $v$ - and $d$ - $H C V$, asterisks); the paths taken by their axons are discussed in the text. Longitudinal veins $L 2-L 5$ are numbered; the nerves found in L3 and the marginal vein (which carries the axons of the numerous marginal bristles) are shown as heavy black lines. Scale bar, $10 \mu \mathrm{m}$ for scanning electron micrographs; $\sim 400 \mu \mathrm{m}$ for the wing; $150 \mu \mathrm{m}$ for the CNS.

times saw a single darkly staining axon plus 1 or 2 faint ones, or a single axon in the medial or lateral tract plus a few in the quite distinct areas occupied by fibers coming from bristles or from the campaniform sensilla of the radial vein (Ghysen, 1978, 1980; Palka et al., 1979). We presume that such cases represent the passage of the microelectrode or the solution it contained through the receptor lymph space of the intended sensillum and into the underlying axon bundle. To avoid any ambiguity, we excluded such fills from our analysis. Had they been accepted, the sample size would have increased by around $50 \%$, but the variability would have increased only slightly.

In some cases, a filled axon could be unambiguously classified as medial or lateral, but some of its branches could not be counted or measured, usually because they were dotted rather than smoothly filled. Therefore, the sample sizes in Figures 5 and 6 are smaller than those of Tables 1 and 2 . In describing the branching patterns, the terminology of Burt and Palka (1982) was used whenever possible.

We applied discriminant function analysis (SPSS version 8.3; see Nie et al., 1975) to determine how well different sensilla within each age group could be distinguished from each other on the basis of the branching patterns of their neurons. Discriminant function analysis is a multivariate technique that assigns individuals characterized by more than one measured attribute into disjoint, predefined groups. In general, it provides (1) tests of significance for differences between the groups; (2) allocation rules for assigning further individuals to one of the groups; and (3) estimates of the probability of correct allocation for each individual. In our case, the individuals were single filled neurons, the multivariate measurements were number and length of specific axonal branches, and the groups were identified sensilla.

The allocation rules for assigning individuals to particular groups can be thought of as straight line boundaries that divide multivariate space into exclusive territories. Each individual is located within some territory that defines the best possible prediction of that individual's group membership. If an additional variable is measured, the analysis may arrive at slightly different boundaries and a more accurate assignment may be achieved. Using only 2 measurements (branch length and branch number for medially projecting axons, and branch number in 2 different locations for laterally projecting axons), we were able to assign nearly $80 \%$ of single axons to their proper sensilla. The probability of correctly assigning an axon to its sensillum ranged from 100 to $44 \%$ (the lateral and medial tracts each contain the axons of 4 sensilla, so chance was $25 \%)$.

For achieving the best separation of individuals into groups, discriminant analysis defines axes (discriminant functions) that have a complex relationship to the original measurements. To plot the boundaries on coordinates that directly represent the original measurements (Figs. 5 and 6), we applied the inverse of the discriminant function matrix to the territorial maps drawn by SPSS for discriminant function space. More detailed information on discriminant analysis can be found in 

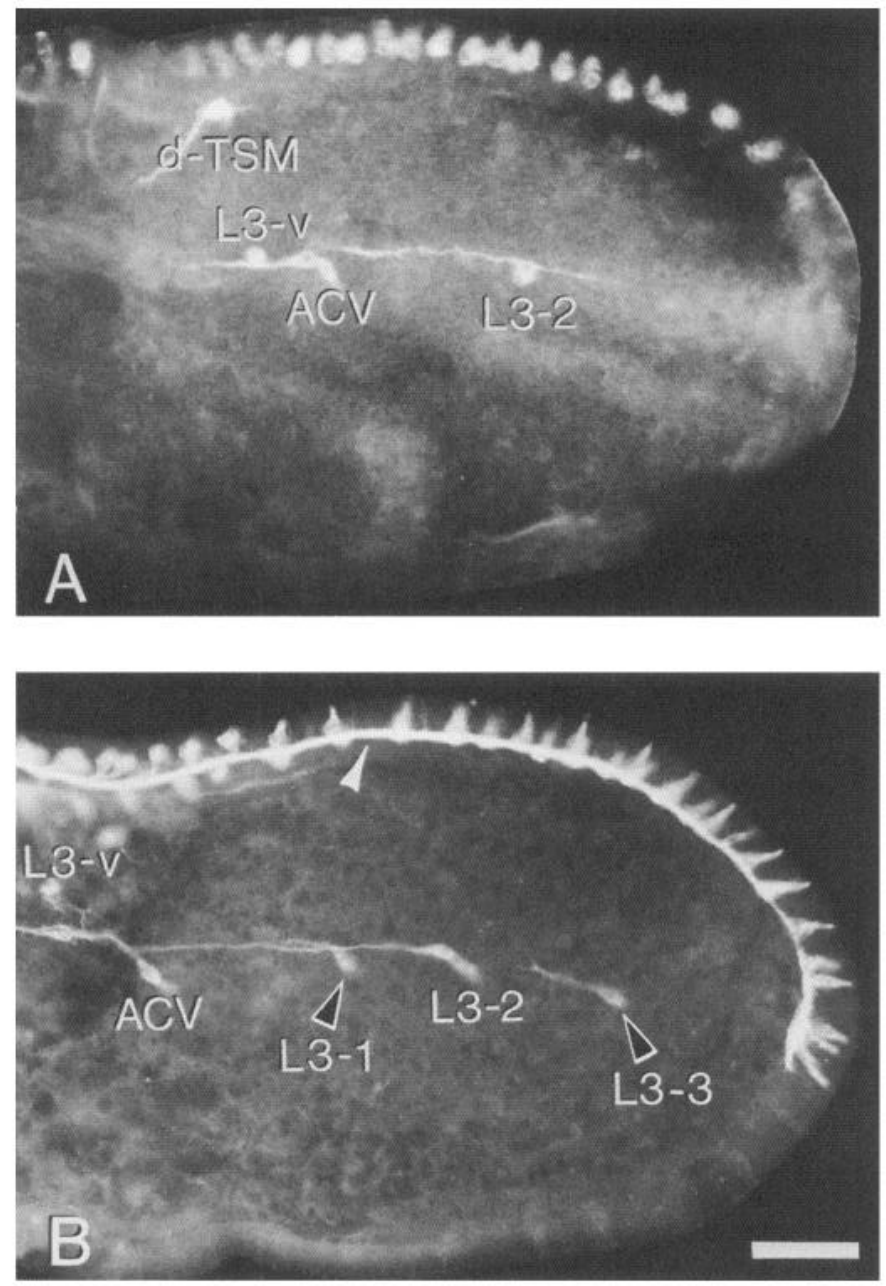

Figure 2. Development of neurons in the wing as revealed by antiHRP indirect immunofluorescence. $A$, Six hour AP: The early neurons $d$-TSM, $A C V$, and $L 3-2$ are shown (GSR is more proximal and out of view to the left); the earliest of the late neurons, $L 3-v$, has also appeared (compare with Table 1). The somata of the bristle neurons along the anterior margin are immunoreactive, but they have not yet produced axons. $B$, Eighteen hour AP: The wing has expanded, and thus the TSM as well as the GSR are now out of view to the left. The late neurons L3-I and L3-3 have appeared (open arrowheads), and their axons can be seen just joining the L3 bundle. The axons of the bristle neurons have grown out to form a thick marginal nerve (solid arrowhead). Scale bar, $50 \mu \mathrm{m}$.

Morrison (1967) and Cooley and Lohnes (1971). Dr. Peter Kareiva of the Department of Zoology, University of Washington, kindly performed this statistical analysis for us.

\section{Results}

\section{Wild-type flies}

The cuticular sense organs of the Drosophila wing are of two types: three rows of mechano- and chemosensory bristles on the leading edge, and dome-shaped campaniform sensilla, responsive to cuticle deformation, distributed along several of the wing veins. In this study we are concerned with the latter class, illustrated in Figure 1. The cuticular component of each campaniform sensillum consists of a central dome surrounded by a circular socket. Its single sensory neuron has a dendrite that attaches to the center of the inner face of the dome and an axon that projects into the CNS. Eight such campaniform sensilla, seemingly identical by scanning electron microscopy, occur in
Table 1. Correlation between the time of development of a wingblade sensillum and the path taken by its axon

\begin{tabular}{|c|c|c|c|c|c|}
\hline \multirow[b]{2}{*}{ Sensillum } & \multicolumn{3}{|c|}{ Hr AP } & \multirow[b]{2}{*}{ Pathway } & \multirow[b]{2}{*}{$N$} \\
\hline & Born & $\begin{array}{l}\text { Anti- } \\
\text { HRP }\end{array}$ & $\begin{array}{l}\text { Axono- } \\
\text { genesis }\end{array}$ & & \\
\hline d-TSM & 0 & 0 & 0 & Medial & $10 / 12$ \\
\hline GSR & 0 & 0 & 0 & Medial & $12 / 12$ \\
\hline $\mathrm{ACV}$ & 0 & 1 & 3 & Medial & $14 / 14$ \\
\hline L3-2 & 0 & 1 & 3 & Medial & $14 / 14$ \\
\hline L3-v & 3 & 4 & 5 & Lateral & $13 / 13$ \\
\hline p-TSM & 8 & 10 & 11 & Lateral & $13 / 13$ \\
\hline L3-1 & 10 & 12 & 13 & Lateral & $16 / 16$ \\
\hline \multirow[t]{2}{*}{ L3-3 } & 10 & 12 & 13 & Lateral & $12 / 12$ \\
\hline & & & & & $104 / 106$ \\
\hline
\end{tabular}

The sensilla are here arranged according to their time of birth, first appearance of HRP-like immunoreactivity, and formation of a $10-\mu \mathrm{m}$-long axon, indicated in hours after pupariation ( $\mathrm{hr}$ AP); the white prepupa stage, lasting only $20-30 \mathrm{~min}$, is taken as $0 \mathrm{hr}$ (data from Murray et al., 1984). The space separates sensilla developing in 2 distinct time periods. Cobalt fills of single axons show that there is a nearly perfect correlation between differentiation during a particular period and projection to a specific pathway.

characteristic locations associated with specific veins on the distal wingblade. Their axons all grow centrally in the same nerve, ramify close to their point of entry into the CNS, and then continue posteriorly in 1 of 2 axon bundles, the medial tract or the lateral tract. Additional campaniform sensilla are found proximally on the wing, in several clusters on the radial vein, and as 2 single sensilla (dorsal and ventral) near the humeral cross vein (d- and v-HCV). None of these match the morphology of the set of 8 sensilla that is the focus of this paper, and all except the d-HCV (see below) appear to use a completely different projection pathway in the CNS (Ghysen, 1978, 1980; Palka et al., 1979).

During the development of the adult wing from the wing imaginal disc of the larva, the various sensory neurons are born, acquire immunoreactivity to anti-HRP antibody (Jan and Jan, 1982), and initiate axonogenesis in a defined sequence (Murray et al., 1984). In particular, the 8 campaniform sensory neurons analyzed here differentiate in 2 waves, as illustrated in Figure 2 and Table 1. The giant sensillum of the radius (GSR), ACV, d-TSM and L3-2 neurons are born prior to $0 \mathrm{hr}$ AP (after pupariation, the beginning of metamorphosis, as defined in Materials and Methods), and show some axon outgrowth by $3 \mathrm{hr}$ AP. The L3-v (ventral), proximal- (p-) TSM, L3-1, and L3-3 neurons are born at $3 \mathrm{hr}$ or later, and initiate axons between 5 and $13 \mathrm{hr}$ AP. We find that all the early-developing sensilla (closed circles in Fig. 1) have branches reaching towards the midline of the CNS and a long axon traveling through the medial tract (solid line in Fig. 1), whereas all the late ones (open circles) have branches confined to the periphery of the neuropil and an axon traveling through the lateral tract (dashed lines). The L3-v sensillum might be considered intermediate in chronology, but its projection is consistently with the late developing group.

Figures 3 (early neurons) and 4 (late neurons) show 3 examples of the central projections of each of the 8 sensilla, and illustrate the data set on which this study is based. The overall difference between the 2 groups is easily seen: Abundant branching reaching the midline and a rather straight, medially located axon characterize all the neurons in Figure 3, while restricted, lateral branching and a curving, more lateral, axon are seen in Figure 4. Table 1 summarizes the paths taken by the axons of our entire sample of 106 neurons filled in wild-type flies. There is a nearly perfect correlation (104/106) between early development and medial projection and late development and lateral projection. 


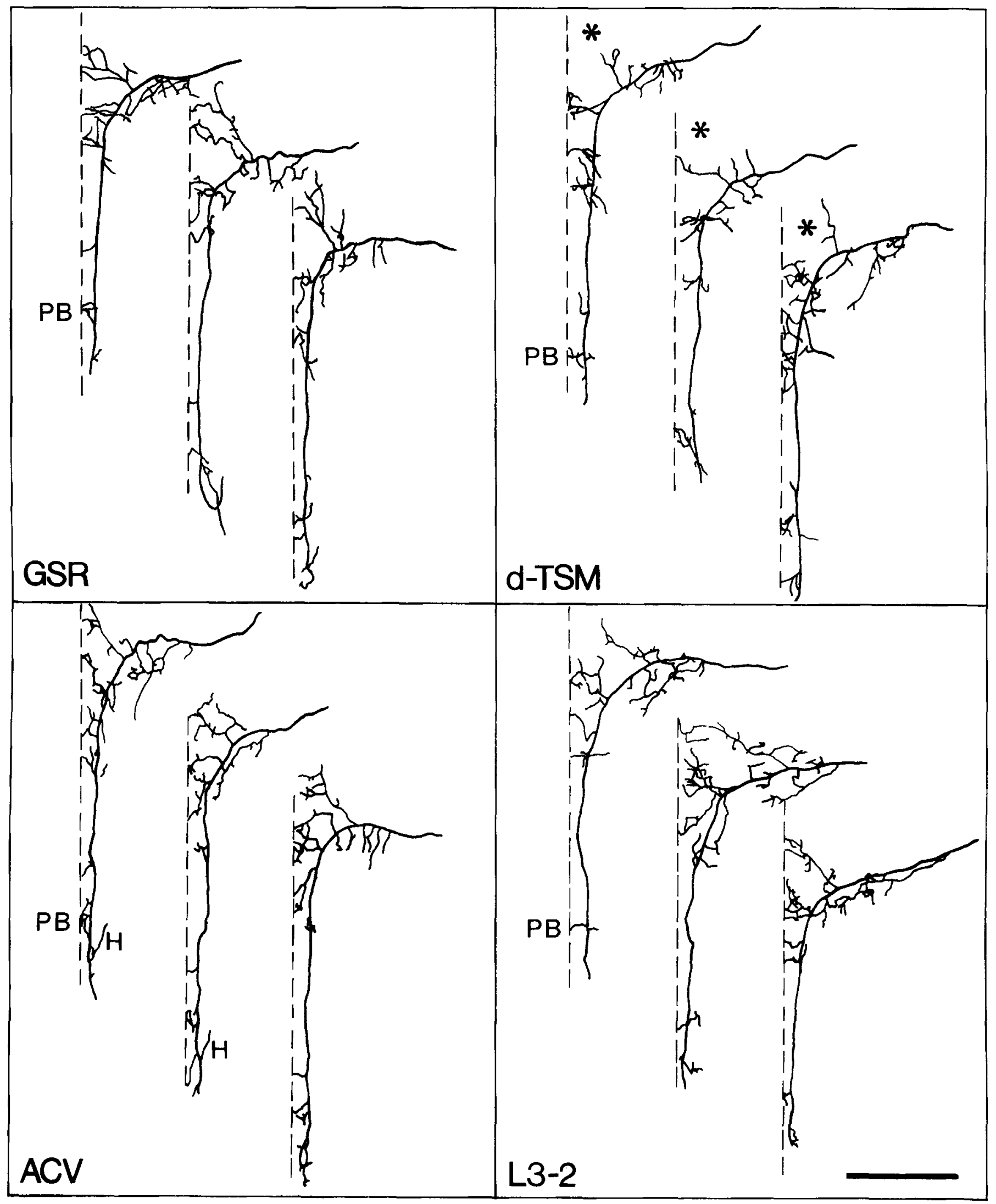

Figure 3. Projections of the 4 early sensilla into the medial tract of the adult CNS. Three examples of each are shown, as viewed with a $50 \times$ oilimmersion lens and drawn with a camera lucida. The approximate midline of the CNS is marked with a dashed line. The branches of the medial axons sometimes extend contralaterally for a short distance. However, inasmuch as many of the sensory fibers from the opposite wing were also filled to create an internal reference within the CNS (see Materials and Methods), the single filled axons drawn here could not be traced reliably past the midline and are arbitrarily shown as terminating there. While the general branching pattern of neurons from all the sensilla is similar, distinctive characteristics can be recognized - for example, the failure of the anteriormost branches of $d$-TSM axons to reach the midline (asterisks). The structures used in the quantitative analysis of Figure 5 are indicated in the $A C V$ drawing: $P B$, a cluster of branches entering a small commissure called the posterior bridge; and $H$, a distinctive hairpin bend by which a descending axon in the medial tract reaches and ascends in the lateral tract. Scale bar, $50 \mu \mathrm{m}$. 
1826

Parka et al.

Vol. 6, No. 6, Jun. 1986

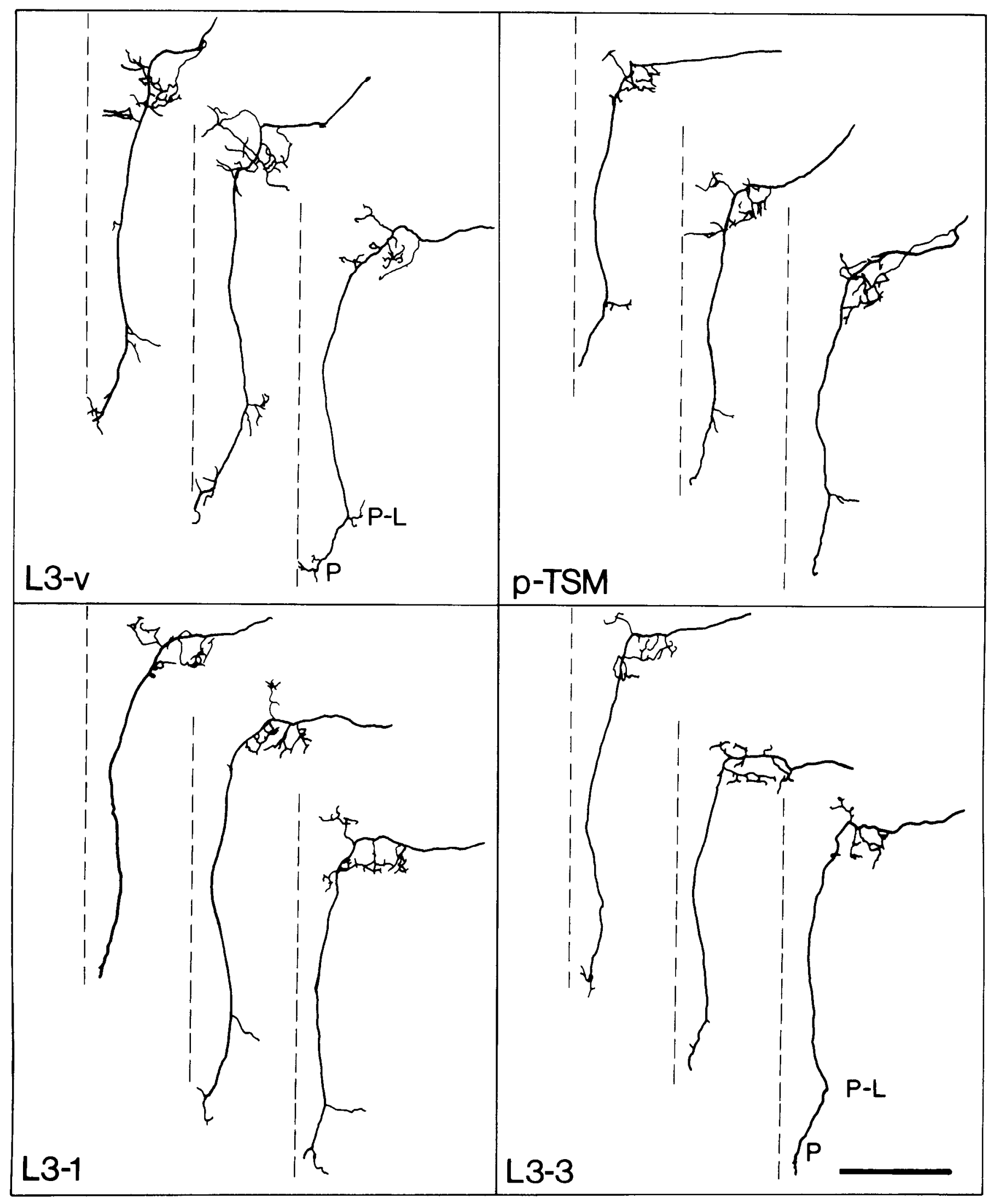

Figure 4. The projections of the 4 late sensilla into the lateral tract of the adult CNS. Drawings prepared as in Figure 3 . No branches reach the
midline. Posterolateral $(P-L)$ and posterior $(P)$ branches were used in the analysis of Figure 6. Scale bar, $50 \mu \mathrm{m}$. 


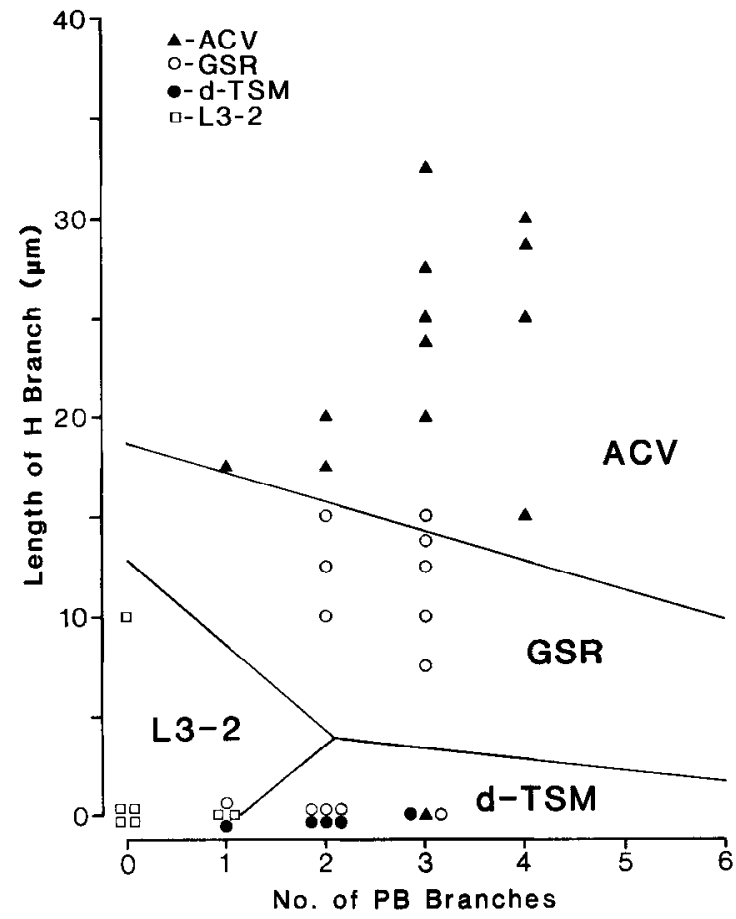

Figure 5. Differences among medially projecting sensilla. In each specimen we counted the number of axon branches entering the posterior bridge (PB in Fig. 3), and measured the length of the distinctive branch that ascends in the lateral tract after making a hairpin turn $(\mathrm{H}$ in Fig. 3). The 4 sensilla are identified by different symbols: $\triangle, A C V ; O, G S R$; - $d-T S M ; \square, L 3-2$. The lines defining the sensillar territories were calculated by discriminant analysis (see Materials and Methods). In this data set, the probabilities of correctly assigning single axons filled in different specimens to their appropriate sensilla are as follows: $\mathrm{ACV}$, 92\%; L3-2, 88\%; d-TSM, 80\%; and GSR, 58\%.

In contrast to the correlation with time of differentiation, there is no simple relationship between the peripheral location of sensillum and the tract followed by its axon. For example, the 2 TSM sensilla (Fig. 1) are less than $20 \mu \mathrm{m}$ apart on the wing, but their axons follow different tracts; reciprocally, the medial and lateral tracts each contain axons from widely separated sensilla. Axons from sensilla on both the dorsal and the ventral surfaces of the wing are found in the lateral tract. Contrary to intuitive expectation, and to a previous report based on fills of a subset of the receptor population (Ghysen, 1980), the axons of sensilla that are neighbors in the periphery do not travel or branch as neighbors in the CNS.

We have asked whether the neurons of the 4 sensilla within each developmental group can be distinguished from one another. The branching patterns are complex and not yet fully characterized, but consistent differences can nevertheless be recognized. For example, the most anterior branches of the d-TSM often fail to reach the midline (Fig. 3, asterisks); the axon of the ACV, which travels down the medial tract, typically forms a hairpin bend and returns for some distance up the lateral tract (Fig. 3, H); the L3-v has substantial posterolateral branching, while the L3-3 has little or none (Fig. 4, P-L); these 2 neurons also differ in the richness of posterior branching (Fig. 4, P); etc. Figures 5 and 6 , in which each sensillum is represented by a different symbol, indicate the correlation among some of these attributes in graphical form, and show that the neurons of the individual sensilla form definable clusters in this "attribute space." The indicated cluster boundaries are based on a discriminant analysis (see Materials and Methods); they represent the statistically most reliable way of predicting the sensillum to

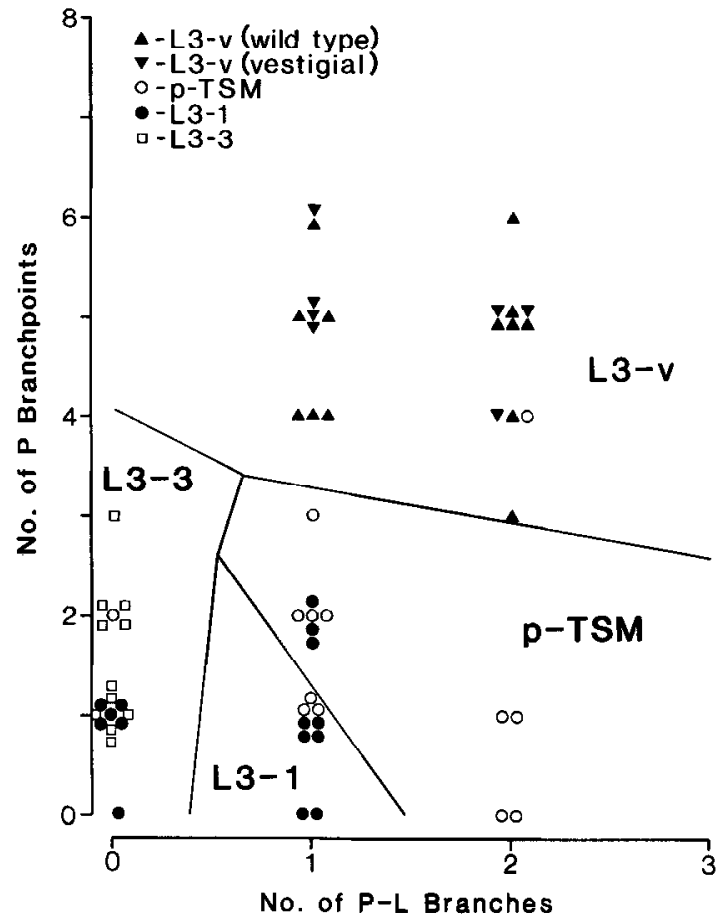

Figure 6. Differences among laterally projecting sensilla. In each specimen we counted the number of posterolateral fibers (P-L in Fig. 4) and the number of branch points in the posterior cluster ( $P$ in Fig. 4). Each sensillum is represented by a different symbol: $\Delta, L 3-v$ (wild type); $\nabla$ L3-v (vestigial); O, p-TSM;,$L 3-1 ; \square, L 3-3$. The lines defining the sensillar territories were calculated by discriminant analysis (see Materials and Methods). In this data set, the probabilities of correctly assigning single axons filled in different specimens to their appropriate sensilla are as follows: L3-3, 100\%; L3-v, 100\%; p-TSM, 54\%; and L3-1, $44 \%$. There is no detectable difference between the $\mathrm{L} 3-\mathrm{v}$ axons of wildtype flies and of vestigial flies.

which an axon with specified branching characteristics belongs. The probability of correctly classifying each of the 4 medial and 4 lateral axons varies from 44 to $100 \%$, with chance being $25 \%$.

\section{Genetic variants}

It is possible to obtain flies with sensilla in altered locations or numbers on the wing by using suitable mutants or by artificial selection for the desired phenotype. Such flies provide "natural experiments" in which the correlation between developmental timing and choice of central tract might be disrupted. However, this correlation has proven to be very robust, surviving all the genetically induced variations in the periphery that we have examined (Table 2). These include (1) differentiation of specific sensilla at normal times but in abnormal locations; (2) failure of specific sensilla to differentiate in otherwise normal wings, as well as failure of receptor-bearing regions of the wing to develop because of extensive degeneration prior to neural differentiation in vestigial mutants (O'Brochta and Bryant, 1983; see Fig. 7B); and (3) addition of supernumerary sensilla in Hairy wing mutants (Fig. 7C).

As shown in Table 2, when a specific sensillum (d-TSM or $\mathrm{ACV}$ ) differentiates in an unusual location, up to $10 \%$ of the total wing length away from its normal position, its axon continues to project into the normal tract. Similarly, when a sensillum is lacking (p-TSM or L3-2), the axons of its neighbors follow their usual tracts. The most extreme example of this stability is seen in vestigial mutants. Their wing phenotype is slightly variable even under uniform rearing conditions, but in the 14 specimens in which we obtained fills the d-TSM, p-TSM, 

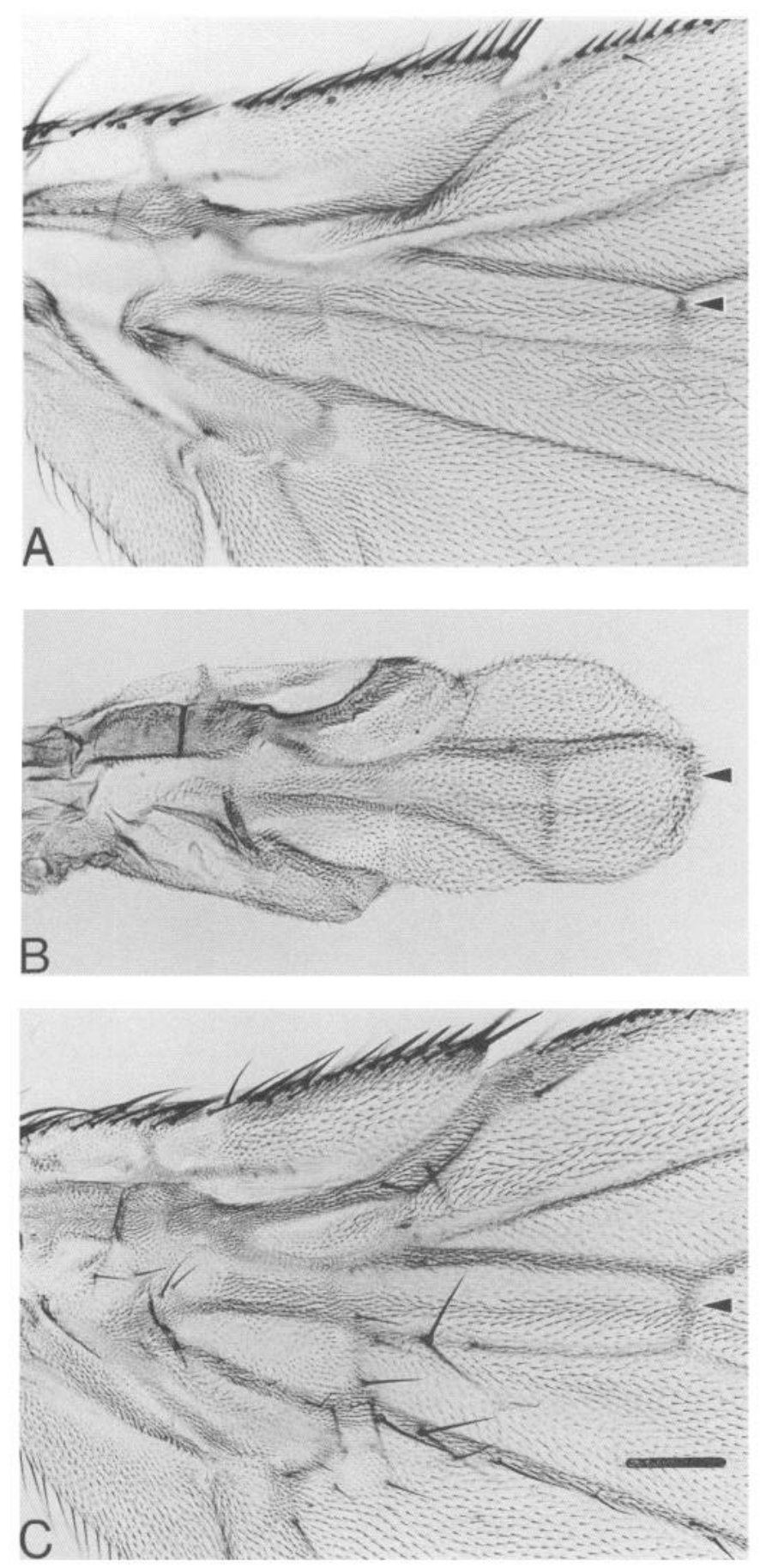

Figure 7. Variant wing phenotypes. A, Wild-type. B, Vestigial ( $v g / v g)$. Only the proximal core of the wing is present; the anterior margin (normally carrying the two TSMs as well as all the innervated bristles), the posterior margin (normally aneural), and the entire blade distal to the ACV (normally carrying L3-1, L3-2, and L3-3) are all absent; thus, of the usual 8 wingblade sensilla, only the GSR, ACV (early), and L3-v (late) remain. $\mathrm{C}$, Hairy wing $(\mathrm{Hw} / \mathrm{Hw})$. Many supernumerary sensilla are present all over the wing, concentrated on but not limited to the veins. Bristles are photographically more conspicuous than campaniform sensilla, but both are abundant. In all specimens, the ACV is marked by an arrowhead. Scale bar, $100 \mu \mathrm{m}$.

L3-1, L3-2, and L3-3 always failed to develop, and only the GSR, ACV, and L3-v were found. The axons of these 3 sensilla followed their usual paths: The early sensilla GSR and ACV projected into the medial tract, and the late sensillum L3-v

\begin{tabular}{|c|c|c|c|c|}
\hline Variant & $\begin{array}{l}\text { Time } \\
\text { of de- } \\
\text { velop- } \\
\text { ment }\end{array}$ & $\begin{array}{l}\text { Pathway } \\
\text { in } \\
\text { normal }\end{array}$ & $\begin{array}{l}\text { Pathway } \\
\text { in } \\
\text { variant }\end{array}$ & $N$ \\
\hline \multicolumn{5}{|l|}{ Location altered } \\
\hline d-TSM moved distally ${ }^{a}$ & Early & Medial & Medial & $1 / 1$ \\
\hline ACV moved proximally ${ }^{b}$ & Early & Medial & Medial & $5 / 5$ \\
\hline \multicolumn{5}{|l|}{ Sensilla lacking } \\
\hline p-TSM lacking, d-TSM filled & Early & Medial & Medial & $7 / 8$ \\
\hline L3-2 lacking, L3-1 filled ${ }^{a}$ & Late & Lateral & Lateral & $1 / 1$ \\
\hline \multicolumn{5}{|l|}{ Vestigial } \\
\hline GSR & Early & Medial & Medial & $5 / 6$ \\
\hline $\mathrm{ACV}$ & Early & Medial & Medial & $4 / 4$ \\
\hline L3-v & Late & Lateral & Lateral & $4 / 4$ \\
\hline \multicolumn{5}{|l|}{ Sensilla added } \\
\hline $\mathrm{L} 3-4^{d}$ & Late & 0 & Lateral & $1 / 1$ \\
\hline \multicolumn{5}{|l|}{ Hairy wing } \\
\hline L2 & Late & 0 & Lateral & $8 / 8$ \\
\hline L3 & Late & 0 & Lateral & $4 / 4$ \\
\hline \multirow[t]{2}{*}{ L4 } & Late & 0 & Lateral & $6 / 6$ \\
\hline & & & & $46 / 48$ \\
\hline
\end{tabular}

" Seen in rare individuals of a line selected for the distal displacement of the d-TSM, with all the other sensilla remaining in their usual locations. Studies on pupal wings in this line showed that the displaced TSM differentiates at the normal, early time. The L3-2, another early sensillum, is sometimes lacking.

${ }^{b} \mathrm{Common}$ in Oregon- $\mathrm{R}$ wild-type stocks, especially when maintained at $25^{\circ} \mathrm{C}$ or above; virtually never seen in Canton $\mathrm{S}$ or Sevelen.

C Common in our line of Sevelen; by up-selection, this phenotype was obtained in $90 \%$ of cases, and by down-selection it was virtually eliminated.

${ }^{d}$ Seen occasionally in a variety of stocks.

- $\mathrm{Hw} / \mathrm{Hw}$.

projected into the lateral tract (Table 2).

Thus, the central axonal pathways of specific sensory neurons are unaffected by translocation of the sensilla, the absence of other neurons whose axons share the same pathway, or the absence of neurons using the alternate pathway. Furthermore, in vestigial flies even the detailed branching characteristics of L3-v, the only late-developing sensillum that remains, match those of the L3-v of wild-type flies (Fig. 6; compare upright and inverted triangles). Evidently, the growth of these axons within the CNS is influenced only minimally, if at all, by that of other axons of the same class.

The supernumerary sensilla of Hairy wing mutants, as well as the single additional sensillum found occasionally in a variety of strains, differentiate later than the normal set (Murray et al., 1984; M. Schubiger, unpublished observations). We filled supernumerary sensilla distributed widely in the region between L3-v and L3-1; they were located on both surfaces of the wing and on 3 different veins, L2 and L3 in the anterior and L4 in the posterior developmental compartment (Garcia-Bellido et al., 1973). Some were examined in the scanning EM; they closely resembled the normal sensilla, except perhaps for a less consistent diameter and a slightly greater tendency to depart from strict radial symmetry. As is shown in Table 2, their axons all $(19 / 19)$ followed the lateral tract, just as the late-differentiating members of the normal set do. This is consistent with the observation that the lateral tract in these flies is unusually thick relative to the medial tract, and much thicker than it is in wildtype flies (see also Ghysen, 1980). Evidently, then, neuronal birth and differentiation in the wing any time after the early wave producing the GSR, ACV, d-TSM and L3-2 is rather strictly correlated with lateral projection within the CNS. 


\section{The $\mathrm{d}-\mathrm{HCV}$ sensillum}

We found a single exception to the early: medial, late: lateral correlation. Both the HCV sensilla (Fig. 1) differentiate early (Murray et al., 1984). The v-HCV projects into a dorsal tract that is quite distinct from the lateral and medial tracts described here (data not shown); it travels with axons from the several clusters of morphologically distinct campaniform sensilla on the radial vein (Cole and Palka, 1982; Ghysen, 1978, 1980; Palka et al., 1979). The axon of the $\mathrm{d}-\mathrm{HCV}$, in contrast, follows the lateral tract (12 out of 12 cases). Thus, it travels with the axons of the late-developing wingblade sensilla in spite of the fact that its cell body differentiates carly, and despite a distinctive morphology that suggests a different physiological function.

\section{Discussion}

\section{Overall organization of the wing campaniform system}

Each of the 8 structurally similar campaniform sensilla of the wingblade projects an axon into either the medial or lateral pathway within the CNS (Fig. 1; Table 1). None of the other wing sensilla share the particular morphology exhibited by these 8 (Cole and Palka, 1982), and only 1 structurally distinctive sensillum (d-HCV) is known to share one of the pathways.

The choice of pathway is highly reliable: Only 2 axons in our sample of 106 fills in wild-type flies did not fit the overall projection pattern. Ghysen (1980), filling axons with HRP introduced by puncturing a vein rather than the dome of a single sensillum, reported slightly higher variability. In both his and our studies, a few axons switching from one pathway to the other, or otherwise behaving aberrantly, were found.

The pathway an axon follows is strictly correlated with the time of birth and differentiation of its neuronal cell body in the wing, even in the face of genetically induced alterations in the number of neurons and in their precise locations (Figs. 1 and 2; Tables 1 and 2). Stability of central projections in the presence of substantial deletions in a sensory population has also been reported for thoracic bristles (Ghysen, 1980). However, contrary to this early report's description of the wingblade projection, which was based on fills of only 3 of the 8 sensilla, we found that the choice of central pathway by these campaniform axons does not correlate with the location of the neuron.

Beyond being distinguished by projecting into either the medial or lateral tract, axons from identified sensilla can be recognized by the details of their branching patterns (Figs. 3-6). Much remains to be done before a full characterization of the neurons can be offered, but even by the few criteria we have used so far, individual neurons within each group of 4 can be classified with reliabilities up to $100 \%$. Whether these distinctive branching patterns constitute a topographically ordered projection within each group is not yet known.

\section{Correlation of developmental history, central projection and function}

We have recently found (Dickinson and Palka, 1985, and unpublished observations) that the early, medially projecting sensilla of wild-type wings respond phasically to mechanical deformation, whereas the late, laterally projecting ones respond tonically. Thus there is at least one physiological difference between the 2 groups, in spite of the uniformity of their cuticular structures as seen by light microscopy or in the scanning EM (Fig. 1). This results in a three-way correlation between time of differentiation, central projection, and physiological function.

The discrete projection of phasic and tonic cells might be considered analogous to the segregation of rapidly and slowly adapting touch units in mammalian somatosensory cortex (Sur et al., 1984), and generally to reflect a functional need for the separate central representation of submodalities. It is at least superficially reminiscent of the cricket cercal mechanosensory system, where the projections of structurally similar filiform hairs that have different directional sensitivities are segregated in the CNS (Bacon and Murphey, 1984). In sharp contrast to the present findings, however, in the cricket system the segregation is a direct consequence of topographic mapping and is apparently not related to developmental timing (Murphey, 1981; Murphey et al., 1983).

\section{Possible causal relations}

At least 3 different, though not mutually exclusive, hypotheses can be proposed to account for the correlation between the time of sensory differentiation in the periphery and the pattern of central projections:

1. The choice of tract depends, at least in part, on a competition for a preferred path (the medial tract), which is won by the early arriving axons; the later ones are then forced into an equally specific, but less attractive, alternative path (the lateral tract). Murphey and Lemere (1984) have demonstrated that competition can influence the projection pattern of insect sensory axons. However, the fact that the axons of the late wing sensilla continue to use the lateral tract even when one or several of the early, medially projecting ones fail to differentiate, and therefore never occupy any sites in the CNS (vestigial, Table 2), argues strongly against a competition hypothesis for explaining the choice of tract in the wing campaniform system. As Figure 6 shows, even the detailed branching pattern of individual neurons seems to be unaffected by the absence of most of the wing's sensory cells.

2. The properties of the CNS change in such a way that only the medial tract can accept axons early in adult development and only the lateral tract can accept them later. Assuming that the time of arrival of an axon in the CNS is systematically related to the time of differentiation of its cell body in the wing, this hypothesis is inconsistent with our observations on the d-HCV sensillum. The d-HCV has a distinctive morphology (Fig. 1) and we consider it to belong to some other functional system than the structurally homogeneous group of sensilla analyzed in this study. Nevertheless, its axon follows the lateral tract. Since the d-HCV is one of the earliest sensilla to differentiatc, the lateral tract appears to be able to accept axons at an early time. Furthermore, it has been reported that at least some supernumerary sensilla located in the proximal regions of the wing in Hairy wing mutants project into the medial tract (Ghysen, 1980). We have not encountered this population (Table 2), but if it is indeed present, the argument against the present hypothesis is strengthened: All the supernumerary sensilla develop later than the normal ones (Murray et al., 1984; M. Schubiger, unpublished observations), so the medial tract must be available to late-arriving axons just as the lateral tract is open to the earlyarriving $\mathrm{d}-\mathrm{HCV}$ axon.

3. Each neuron independently recognizes markers associated with specific pathways within the already existing CNS (Ghysen, 1980; Goodman et al., 1982), and developmental timing is related to the acquisition of particular physiological and pathway recognition mechanisms by neurons, rather than having a direct causal role in the guidance of axons through the CNS. We consider this to be the most attractive hypothesis, especially in view of the demonstration of physiological differences between medially and laterally projecting sensilla (Dickinson and Palka 1985).

The relationship we have observed between time of development and central projection and/or physiological function is not unique. Along the margin of the wing, the chemosensory bristle neurons appear to differentiate early and the purely mechanosensory ones as a second wave (Murray et al., 1984). Further, in the Drosophila retina, the 8 receptor cells comprising each ommatidium are born and differentiate in 2 waves (Ready et 
al., 1976), and in the cat retina, different classes of ganglion cells develop in successive waves (Walsh and Polley, 1985; Walsh et al., 1983). It may well be a widespread principle, related in some way to the mechanisms that generate neuronal diversity, that functionally distinctive cells of the same general class have different temporal histories.

Our experiments, of course, do not address the question of how specific pathways within the CNS might be recognized by growing axons. We only note that, on the basis of the regions in which they branch, the longitudinal pathways that they follow, and at least one physiological attribute, the early sensilla differ as a group from the late ones. According to the models suggested by Zipser et al. (1983) and Kotrla and Goodman (1984), the members of each group would be expected to share some distinctive antigen(s) associated with their function and/ or the mechanism of their selective fasciculation. The discovery of antibodies that reveal such group-characteristic antigens would provide striking support for these models.

\section{References}

Bacon, J. P., and J. S. Altman (1977) A silver intensification method for cobalt-filled neurons in wholemount preparations. Brain Res. 138: 359-363.

Bacon, J. P., and R. K. Murphey (1984) Receptive fields of cricket giant interneurones are related to their dendritic structure. J. Physiol. (Lond.) 352: 601-623.

Burt, R., and J. Palka (1982) The central projections of mesothoracic sensory neurons in wild-type Drosophila and bithorax mutants. Dev. Biol. 90: 99-109.

Cole, E. S., and J. Palka (1982) The pattern of campaniform sensilla on the wing and haltere of Drosophila melanogaster and several of its homeotic mutants. J. Embryol. Exp. Morph. 71: 41-61.

Cooley, W. W., and P. R. Lohnes (1971) Multivariate Data Analysis, Wiley, New York.

Dickinson, M. H., and J. Palka (1985) Physiological characteristics of sensory neurons of Drosophila wing correlate with central projections and developmental timing. Soc. Neurosci. Abstr. 11: 164.

Garcia-Bellido, A., P. Ripoll, and G. Morata (1973) Developmental compartmentalization of the wing disk of Drosophila. Nature New Biol. 245: 251-253.

Ghysen, A. (1978) Sensory neurons recognize defined pathways in Drosophila central nervous system. Nature 274: 869-872.

Ghysen, A. (1980) The projection of sensory neurons in the central nervous system of Drosophila: Choice of the appropriate pathway. Dev. Biol. 78: 521-541.

Goodman, C. S., J. A. Raper, R. K. Ho, and S. Chang (1982) Pathfinding by neuronal growth cones in grasshopper embryos. In Developmental Order: Its Origin and Regulation, S. Subtelny and P. G. Green, eds., pp. 275-316, Alan R. Liss, New York.

Jan, L. Y., and Y. N. Jan (1982) Antibodies to horseradish peroxidase as specific neuronal markers in Drosophila and in grasshopper embryos. Proc. Natl. Acad. Sci. USA 79: 2700-2704.

Johnson, S. E., and R. K. Murphey (1985) The afferent projection of mesothoracic bristle hairs in the cricket, Acheta domesticus. J. Comp. Physiol. A 156: 369-379.
Kotrla, K. J., and C. S. Goodman (1984) Transient expression of a surface antigen on a small subset of neurones during embryonic development. Nature 311: 151-153.

Lindsey, D. L., and E. H. Grell (1968) Genetic Variations of Drosophila Melanogaster. Carnegie Institution of Washington, Pub. 627, Washington, DC.

Morrison, D. F. (1967) Multivariate Statistical Methods, McGrawHill, New York.

Murphey, R. K. (1981) The structure and development of a somatotopic map in crickets: The cercal afferent projection. Dev. Biol. 88 . 236-246.

Murphey, R. K., and C. A. Lemere (1984) Competition controls the growth of an identified axonal arborization. Science 224: 1352-1355.

Murphey, R. K., A. Jacklet, and L. Schuster (1980) A topographic map of sensory cell terminal arborizations in the cricket CNS: Correlation with birthday and position in a sensory array. J. Comp. Neurol. 191: 53-64.

Murphey, R. K., S. E. Johnson, and D. S. Sakaguchi (1983) Anatomy and physiology of supernumerary cercal afferents in crickets: Implications for pattern formation. J. Neurosci. 3: 312-325.

Murray, M. A., M. Schubiger, and J. Palka (1984) Neuron differentiation and axon growth in the developing wing of Drosophila melanogaster. Dev. Biol. 104: 259-273.

Nie, N., C. Hull, J. Jenkins, K. Steinbrenner, and D. Best (1975) Statistical Package for Social Sciences, 2nd Ed., McGraw-Hill, New York.

O'Brochta, D. A., and P. J. Bryant (1983) Cell degeneration and elimination in the imaginal wing disc, caused by the mutations vestigial and ultravestigial of Drosophila melanogaster. Wilh. Roux Arch. 192: 285-294.

Palka, J., P. A. Lawrence, and H. S. Hart (1979) Neural projection patterns from homeotic tissue of Drosophila studied in bithorax mutants and mosaics. Dev. Biol. 69: 549-575.

Ready, D. F., T. E. Hanson, and S. Benzer (1976) Development of the Drosophila retina, a neurocrystalline lattice. Dev. Biol. 53: 217240.

Strausfeld, N. J. (1976) Mosaic organizations, layers, and visual pathways in the insect brain. In Neural Principles in Vision, F. Zettler and R. Weiler, eds., pp. 245-279, Springer-Verlag, Berlin.

Sur, M., J. T. Wall, and J. H. Kaas (1984) Modular distribution of neurons with slowly adapting and rapidly adapting responses in area $3 \mathrm{~b}$ of somatosensory cortex in monkeys. J. Neurophysiol. 51: 724 744.

Thurm, U., and J. Kuppers (1980) Epithelial physiology of insect sensilla. In Insect Biology in the Future, "VBW 80 ," M. Locke and D. S. Smith, eds., pp. 735-763, Academic, New York.

Walsh, C., and R. W. Guillery (1984) Fibre order in the pathways from the eye to the brain. Trends Neurosci. 7: 208-211.

Walsh, C., and E. H. Polley (1985) The topography of ganglion cell production in the cat's retina. J. Neurosci. 5: 741-750.

Walsh, C., E. H. Polley, T. L. Hickey, and R. W. Guillery (1983) Generation of cat retinal ganglion cells in relation to central pathways. Nature 302: 611-614.

Wigston, D. J., R. L. Ellison, and J. Palka (1984) Choice of central pathways by sensory neurons in Drosophila. Soc. Neurosci. Abstr. 10: 139.

Zipser, B., R. Stewart, T. Flanagan, M. Flaster, and E. R. Macagno (1983) Do monoclonal antibodies stain sets of functionally related leech neurons? Cold Spring Harbor Symp. Quant. Biol. 48: 551-556. 